\title{
Dual sensing-actuation artificial muscle based on polypyrrole-carbon nanotube composite
}

\author{
J. Schumacher, ${ }^{\mathrm{a}, \mathrm{b}}$, Toribio F. Otero ${ }^{\mathrm{b}}$, Victor H. Pascual ${ }^{\mathrm{b}}$ \\ ${ }^{a}$ Arquimea Ingeniería, Margarita Salas 10, 28919, Leganés, Spain; ${ }^{b}$ Laboratory of Electrochemistry \\ and Intelligent Materials, Technical University of Cartagena, ETSII, Campus Alfonso XIII, 30203, \\ Cartagena, Spain.
}

\begin{abstract}
Dual sensing artificial muscles based on conducting polymer are faradaic motors driven by electrochemical reactions, which announce the development of proprioceptive devices. The applicability of different composites has been investigated with the aim to improve the performance. Addition of carbon nanotubes may reduce irreversible reactions. We present the testing of a dual sensing artificial muscle based on a conducting polymer and carbon nanotubes composite. Large bending motions (up to 127 degrees) in aqueous solution and simultaneously sensing abilities of the operation conditions are recorded. The sensing and actuation equations are derived for incorporation into a control system.
\end{abstract}

Keywords: self-sensing actuator, dual sensing-actuation, sensing molecular conformational machines, proprioception, conducting polymer composite, carbon nanotubes, faradaic processes

\section{INTRODUCTION}

Smart material actuators can be engineered for fluidic pumps ${ }^{1-4}$, valves ${ }^{5,6}$, soft robots ${ }^{7,8}$ and adaptive optics ${ }^{9,10}$. In those applications the use of external sensors is often not practical and self-sensing actuators are desired to minimize the required system elements.

Self-sensing electromechanical actuators such as dielectric elastomers ${ }^{11-13}$, IPMCs ${ }^{14,15}$, shape memory alloys ${ }^{16}$, piezoelectric ${ }^{17-19}$ and magnetostrictive materials ${ }^{20}$ function as one sensor (resistance, capacitance) during actuation. Both, sensor and actuator are spatially separated in the device. In contrast, in electro-chemo-mechanical actuators based on conducting polymers ${ }^{21-23}$ several sensors and one actuator work simultaneously in a physically uniform device driven by the electrochemical reaction of the constitutive material (conducting polymer). Only two connecting wires contain, at any time, driving and sensing signals: current and charge control the muscle movement rate and position and the muscle potential or consumed energy defines the working mechanical, thermal or electrical conditions ${ }^{22,24}$. Actuation and sensing mechanisms are parallel to vertebrate haptic muscles and motor neurons originating proprioception. Hence, the latter inherits the potential to replicate biological systems with the lowest complexity level.

Besides conducting polymers, electrochemical reactions from carbon nanotubes ${ }^{25,26}$ or graphenes ${ }^{27}$ also sense the working energetic conditions and, hence, are suitable materials for the construction of sensing-motors. Conducting polymer carbon nanotube composites are expected to have similar behaviour. The actuation performance of actuators based on composites material of conducting polymer and carbon nanotubes has been explored extensively. The submission to square potential waves at different frequencies ranging from $0.017 \mathrm{~Hz}^{28}$ up to $200 \mathrm{~Hz}^{29}$ revealing longterm actuation up to 14.000 cycles at $1 \mathrm{~Hz}^{28}$. The strain was found to be dependent on the frequency with maximum strains at low frequencies ${ }^{29}$. Actuation tests of a layered PAN/CNT/PPY composite muscle were conducted with pulsed current $(1 / 60 \mathrm{~Hz})$ for electrochemical stimulation showing low actuation strains but superior actuation under load ${ }^{30}$. The 
submission to potential cycles at a specific sweep rate revealed the presence of oxidation and reduction processes ${ }^{28,31-34}$ resulting in large bending displacements ${ }^{33}$.

Here, we present the dual sensing and actuation performance of artificial muscles based on a conducting polymer and carbon nanotubes composite operated with triangular potential wave signals at different frequencies. The motion control is investigated and non-linear effects such as hysteresis are modelled. Furthermore, the sensing equation is derived.

\section{METHODOLOGY}

\subsection{Materials}

Pyrrole (Fluka) was vacuum-distilled prior use and stored at $-10^{\circ} \mathrm{C}$. Sodium perchlorate (NaClO4) (Sigma-Aldrich), multi-walled carbon nanotubes (MWCNT) (Sigma-Aldrich, purity $>90 \%$, outer diameter 10-15 nm, inner diameter 2-6 $\mathrm{nm}$, length $0.1-10 \mu \mathrm{m}$ ) and sodium dodecylbenzenesulfonate (NaDBS) (Sigma-Aldrich) were used as supplied. Milli-Q water was used for all aqueous solutions.

\subsection{Equipment and Experimental Conditions}

For the electrochemical experiments and electrochemical polymerization an Autolab potentiostat/galvanostat PGSTAT302 (NOVA 1.11 software) was used with a three electrode configuration, an $\mathrm{Ag} / \mathrm{AgCl}(3 \mathrm{M} \mathrm{KCl})$ reference electrode (RE, Metrohm) and stainless steel counter electrodes (CE). All experiments were performed at room temperature.

A Sartorius SC2 balance $( \pm 0.1 \mathrm{mg})$ was used to determine the total mass of the electropolymerized films via the difference between coated and uncoated working electrode. The thickness of the composite film and the actuator was measured with a Helios Preisser digital micrometer $( \pm 0.1 \mu \mathrm{m})$. The bending displacements of the bilayer muscle were quantified from the video-frames recorded by a 1080p high-resolution camera using a Matlab program.

\subsection{Electropolymerisation and fabrication}

The PPy-DBS-MWCNT films were synthesised by potential steps $(-0.2 \mathrm{~V}$ for $1 \mathrm{~s}, 0.8 \mathrm{~V}$ for $3600 \mathrm{~s})$ in monomeric aqueous solution $(0.03 \mathrm{M}$ NaDBS, $0.1 \mathrm{M}$ pyrrole, $0.1 \mathrm{wt} \% \mathrm{MWCNT})$ using a three-electrode configuration where three stainless steel sheets were parallel assembled at a distance of $1 \mathrm{~cm}$. The outer ones were applied as counter electrode (CE) and the central one as working electrode (WE). The deposited PPy-DBS-MWCNT films on the WE were washed in water to remove the excess of NaDBS, dried in air and removed from the stainless steel plate. The thickness and the weight of the films were $48.6 \mu \mathrm{m}$ and $9.3 \mathrm{mg}$, respectively. The film was attached to a passive layer (tape, duo Fixo) and cut into $20 \times 3 \mathrm{~mm}$ longitudinal strips with a total thickness of $116 \mu \mathrm{m}$. The fabrication process has been described preciously ${ }^{33}$. Finally, a strip of paint was transversally drawn around the bilayer at $1.5 \mathrm{~mm}$ from the top to avoid the electrolyte capillarity towards the electrical contact when immersed in electrolyte.

\subsection{Electro chemo dynamical measurements}

For measurements, each bilayer actuator was fixed in an electrical contact and used as WE in an electrochemical cell with fresh $0.1 \mathrm{M} \mathrm{NaClO}_{4}$ aqueous electrolyte solution (Figure 1). The actuator was submitted to consecutive potential sweeps with sweep rates ranging from $0.5 \mathrm{mVs}^{-1}$ to $100 \mathrm{mVs}^{-1}$ with a potential range of -1.2 to $0.7 \mathrm{~V}$ equivalent to triangular potential waves with frequencies of $0.0001 \mathrm{~Hz}$ to $0.0263 \mathrm{~Hz}$. The consumed charge was simultaneously calculated by the potentiostat. The reversible bending movements described by the actuator were video recorded in parallel to the potential cycling. 


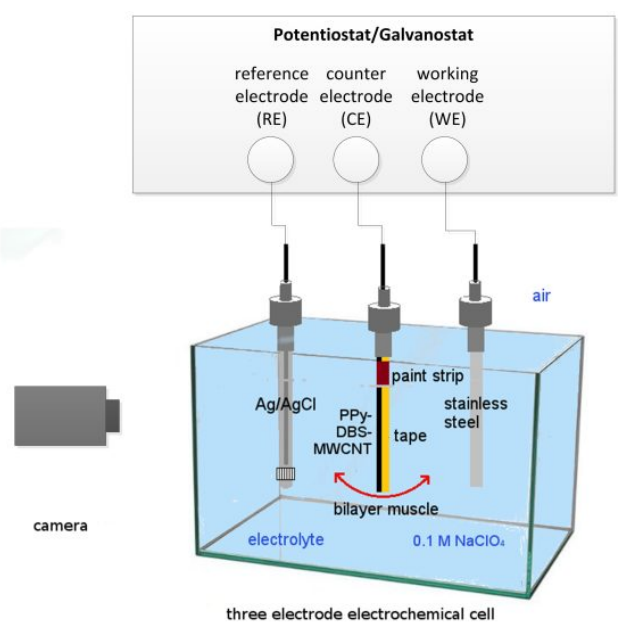

Figure 1 Experimental set-up for electro-chemo-dynamical characterization

\section{RESULTS AND DISCUSSION}

\subsection{Electroactivity}

Figure 2 shows a typical voltammetric response when the actuator was submitted to potential sweeps at $10 \mathrm{mVs}^{-1}$ (triangular waves at $0.0026 \mathrm{~Hz}$ ) indicating the electroactivity of the composite layer. The actuator shows a similar electrochemical behavior to those attained from previous investigations of conducting polymer carbon nanotube composites revealing the presence of oxidation and reduction maxima ${ }^{28,31-35}$.
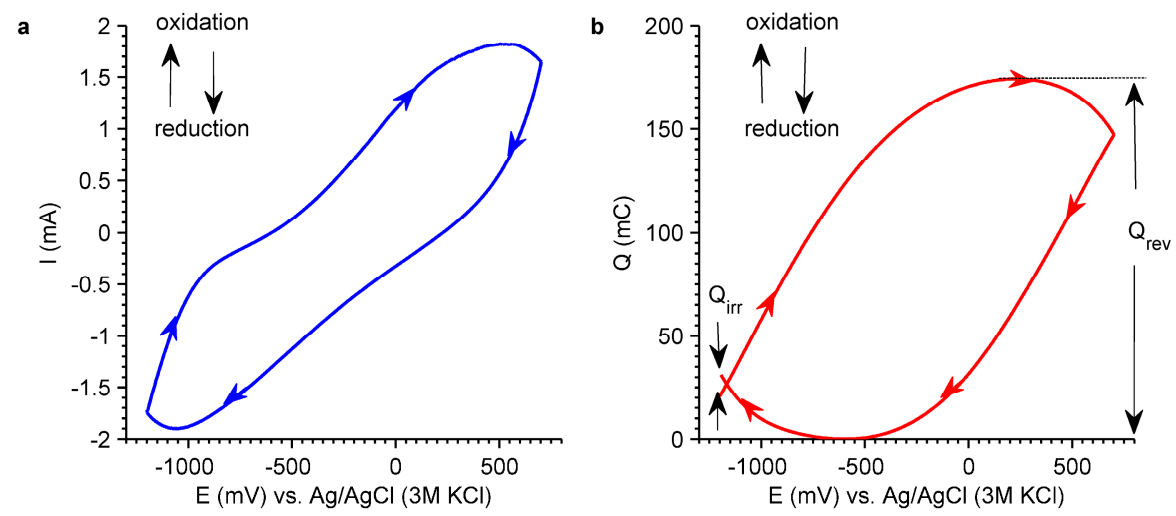

Figure 2 Voltammetric (a) and coulovoltammetric (b) responses attained from PPy-DBS-MWCNT bilayer at $10 \mathrm{mVs}^{-1}$ in $0.1 \mathrm{M}^{-}$ $\mathrm{NaClO}_{4}$ aqueous solution using a potential range from -1200 to $700 \mathrm{mV}$ (vs. $\mathrm{Ag} / \mathrm{AgCl}$ )

The integration of the voltammetric (current-potential, I-E) response (Figure 2a) gives the concomitant coulovoltammetric (charge-potential, Q-E) response depicted by Figure $2 \mathrm{~b}$. The coulovoltammetric response displays the charges consumed by the actuator during the redox reaction. Positive charge increments are given by oxidation charges and reduction charges give negative charge increments. During actuation the actuator consumes reversible charges $\left(\mathrm{Q}_{\text {rev }}\right)$ giving a closed coulovoltammetric loop: oxidation charges equal reduction charges. A small irreversible charges $\left(\mathrm{Q}_{\text {irr, }}\right.$ 
Figure $2 \mathrm{~b}$ ) is consumed by simultanous irreversible reactions such as the hydrogen evolution from the HDBS component 36. Irreversible charges do not contribute to the actuation and can degrade the material electroactivity. The presence of CNT decreases this irreversible charge ${ }^{33,36}$.

\subsection{Range of movement (angular displacement) at different frequencies}

The actuator shows reversible bending movements at all tested frequencies shown in Figure 3a. The angular displacement (described angle) increases at decreasing frequency up to $127^{\circ}$ for $0.0003 \mathrm{~Hz}$. Lower frequencies mean longer oxidation/reduction times with the consumption of increasing reaction charges which means higher amounts of exchanged ions according to the Faraday's laws and solvent for osmotic balance resulting bigger volume changes and thus higher angular displacements of the muscle tip.

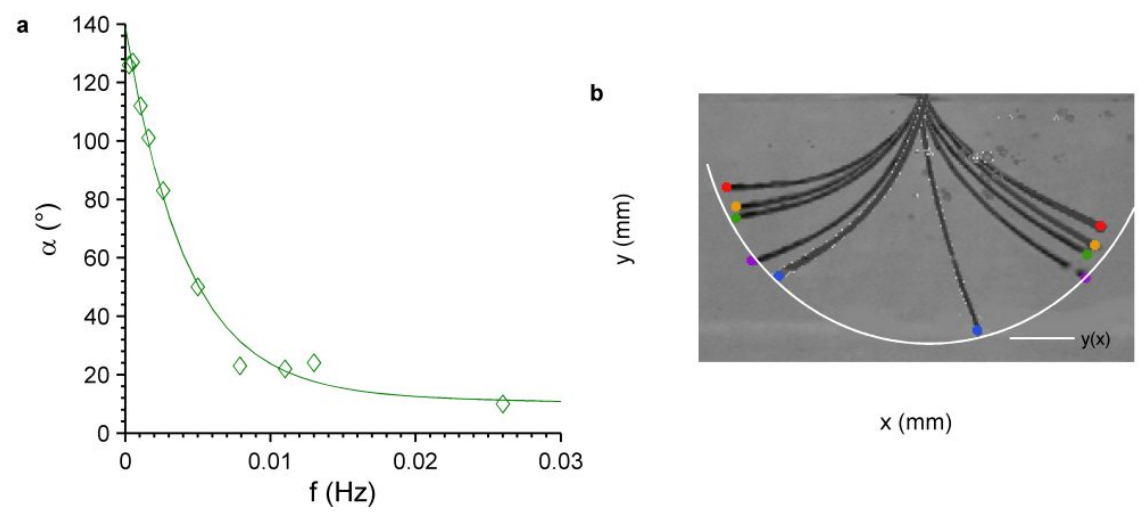

Figure 3 (a) total angular bending range at different frequencies in $0.1 \mathrm{M} \mathrm{NaClO}_{4}$ aqueous solution (b) x-displacement vs ydisplacement at $0.003 \mathrm{~Hz}$ (red), $0.005 \mathrm{~Hz}$ (yellow), $0.0011 \mathrm{~Hz}$ (purple) and $0.0016 \mathrm{~Hz}$ (blue)

The experimental results can be adjusted by addition of two exponential functions:

$$
\alpha=a e^{b f}+c e^{d f}
$$

where $\mathrm{a}=126^{\circ}, \mathrm{b}=-240 \mathrm{~s}, \mathrm{c}=13^{\circ}, \mathrm{d}=-6.81 \mathrm{~s}$ and the correlation coefficient $\mathrm{r}^{2}=0.99$. By extrapolation of Eq. 1 the theoretical limit of the motion range of the actuator can be calculated when the frequency f approaches $0(f=0)$.

$$
\alpha_{\text {max }}=a+c=139^{\circ}
$$

Hence, the maximum motion range of the actuator is $139^{\circ}$.

\subsection{Bending trajectory}

The bending trajectory of the actuator tip is illustrated in Figure $3 \mathrm{~b}$ for low frequencies $(<=0.0026 \mathrm{~Hz})$. In the tested vertical configuration the actuator is able to bend clockwise and anticlockwise from the initial vertical position. The tip moves on an elliptical arc which center is shifted on the y-axis by $h$.

$$
\begin{aligned}
& \left(\frac{x}{k}\right)^{2}+\left(\frac{y-h}{l}\right)^{2}=1 \\
& y(x)=-l \cdot \sqrt{1-\left(\frac{x}{k}\right)^{2}}+h
\end{aligned}
$$

where $\mathrm{l}=150 \mathrm{~mm}, \mathrm{~h}=150 \mathrm{~mm}$ and $\mathrm{k}=32 \mathrm{~mm}$. 
Figure $3 \mathrm{~b}$ reveals that the bending range is not symmetrical. The actuator bends more to the left side than to the right referred to the vertical position of the actuator. For the integration of the actuator into a device this has to be taken into account.

\subsection{Position control}

PPy-DBS-CNT actuators are faradaic motors ${ }^{33}$. Hence, the tip position of the bending actuator is controlled by the consumed charge. Figure 4a displays the tip position by the angle with reference to the straight vertical position $\left(0^{\circ}\right)$. Positive angles indicate clockwise bending movement and negative angles indicate anticlockwise bending movement.
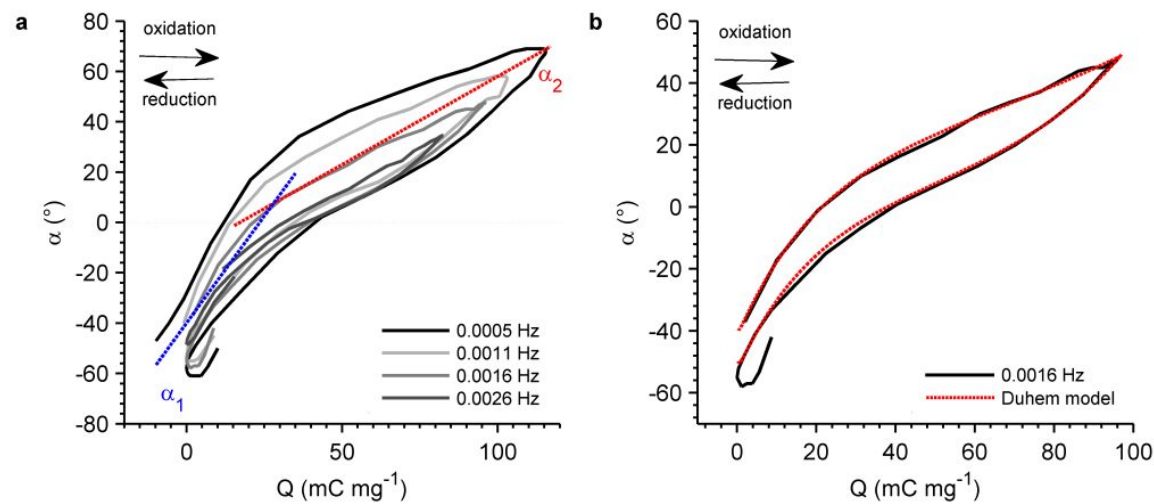

Figure 4 (a) coulodynamic response for low frequencies with two linear sections indicated for $0.0005 \mathrm{~Hz}\left(2 \mathrm{mVs}^{-1}\right)$ (b) empirical hysteresis loop at $0.0016 \mathrm{~Hz}\left(6 \mathrm{Vms}^{-1}\right)$ of the actuator and hysteresis loop of Duhem model

In average, and taking into account hysteresis effects due to the asymmetry of the osmotic and electroosmotic effects driving the exchange of solvent that follows the ion exchange ${ }^{37}$, linear correlations exist between the angle and the charge for the whole potential range at each studied frequency. The coulodynamic responses show two linear sections, indicating the existence of two different driving reactions each related to one of the electroactive components, PPy and CNT ${ }^{33}$. Whatever the experimental frequency, the angular displacement is under linear control of the consumed electrical charge, as it corresponds to a Faradaic motor ${ }^{38}$ :

$$
\begin{aligned}
& \alpha_{1}=\alpha_{0_{1}}+\mathrm{k}^{\prime}{ }_{1} \cdot \mathrm{Q} \\
& \alpha_{2}=\alpha_{0_{2}}+\mathrm{k}^{\prime}{ }_{2} \cdot \mathrm{Q}
\end{aligned}
$$

with $\mathrm{k}_{1}{ }_{1}=0.7^{\circ} \mathrm{mC}^{-1}, \mathrm{k}_{2}{ }_{2}=1.5^{\circ} \mathrm{mC}^{-1}$ and $\alpha_{10}$ and $\alpha_{20}$ being listed in Table 1 .

Table 1 specific values of $\alpha_{0_{1}}$ and $\alpha_{0_{2}}$ for low frequencies

\begin{tabular}{|c|c|c|}
\hline $\begin{array}{c}\text { Frequency } \\
\mathrm{Hz}\end{array}$ & $\begin{array}{c}\alpha_{0_{1}} \\
\mathrm{o}\end{array}$ & $\begin{array}{c}\alpha_{0_{2}} \\
\mathrm{o}^{\circ}\end{array}$ \\
\hline 0.0003 & 40 & 12 \\
\hline 0.0005 & 38 & 14 \\
\hline 0.0011 & 42 & 20 \\
\hline 0.0016 & 45 & 24 \\
\hline
\end{tabular}

Each constant $\mathrm{k}$ describes the angle-charge relationship (angle described per unit of specific charge) independent of the frequency, being a characteristic of every electroactive material-electrolyte system. In contrast the initial angle $\alpha_{0}$ is frequency dependent resulting in a parallel shift of the linear dependence (Eq. 5 and Eq. 6). 
The consumption of irreversible reduction charges, in Figure 4a negative charges, result in an angular shift at the end of each cycle (creeping effect) and a complex control at the movement limit (maximum bending position) to the left (hook shape) side.

Figure 4a shows that large hysteresis effects (different angular position for the same oxidation state of the material: same charge) are present for lower frequencies, and in an open loop configuration, the hysteresis is $26 \%$ of the total displacement at $0.0005 \mathrm{~Hz}$ of the actuator resulting in inaccuracy in positioning control. In addition the hysteresis curves are frequency dependent. Hence, for the sensorless position control of the PPy-DBS-CNT actuator a more accurate mathematical model is required. The Preisach model ${ }^{39-41}$, the Prandtl-Ishlinskii model ${ }^{42,43}$ or the Maxwell model ${ }^{44,45}$ are widely used for other smart material based actuator to describe hysteresis effects. For trilayer conducting polymer actuators the Duhem model was established ${ }^{46}$.

The Duhem model can be described by:

$$
\frac{d \alpha}{d t}=p\left|\frac{d Q}{d t}\right|[f(Q)-\alpha]+\frac{d Q}{d t} g(Q)
$$

where $f(Q)$ and $g(Q)$ are the characteristic functions of the hysteresis model, $Q(t)$ is the input signal and $\alpha(t)$ is the output signal. The characteristic functions $\mathrm{f}(\mathrm{Q})$ and $\mathrm{g}(\mathrm{Q})$ can be obtained with mathematical functions which describe the ascending (oxidation) and descending (reduction) branches and the weighing factor $\mathrm{p}^{46}$. For the modeling of the hysteresis the complex bending (hook shape, Figure 4) are neglected. Here, the two branches are described with double exponential functions determined by standard least square technique.

Defining the consumed charge $\mathrm{Q}$ as input of the hysteresis results in

$$
Q(t)= \begin{cases}I \cdot t & \text { oxidation } \\ -I \cdot t & \text { reduction }\end{cases}
$$

then,

$$
\frac{d Q(t)}{d t}= \begin{cases}I & \text { oxidation } \\ -I & \text { reduction }\end{cases}
$$

where I is the current flowing during reduction and oxidation of the PPy-DBS-CNT film. Figure 4b shows the simulated hysteresis loop from the Duhem hysteresis model of Eq. 7 and proofs that the Duhem model is an effective method to model the dynamic hysteresis effects of PPy-DBS-MWCNT actuators. This hysteresis model can be established with series connection or feedback connection. In contrast to the presented hysteresis model by Wang ${ }^{46}$, here, the hysteresis is frequency dependent. Future work will require a revision of the model and establish a rate depend hysteresis model.

\subsection{Sensing}

Figure 5 shows the charge evolution of the PPy-DBS-CNT actuator operated with triangular waves at different frequencies $\mathrm{f}$. The reversible charge decreases with the frequency caused by the limitation of ion transport across the swelling/shrinking film, from or towards the solution, at higher frequencies. 

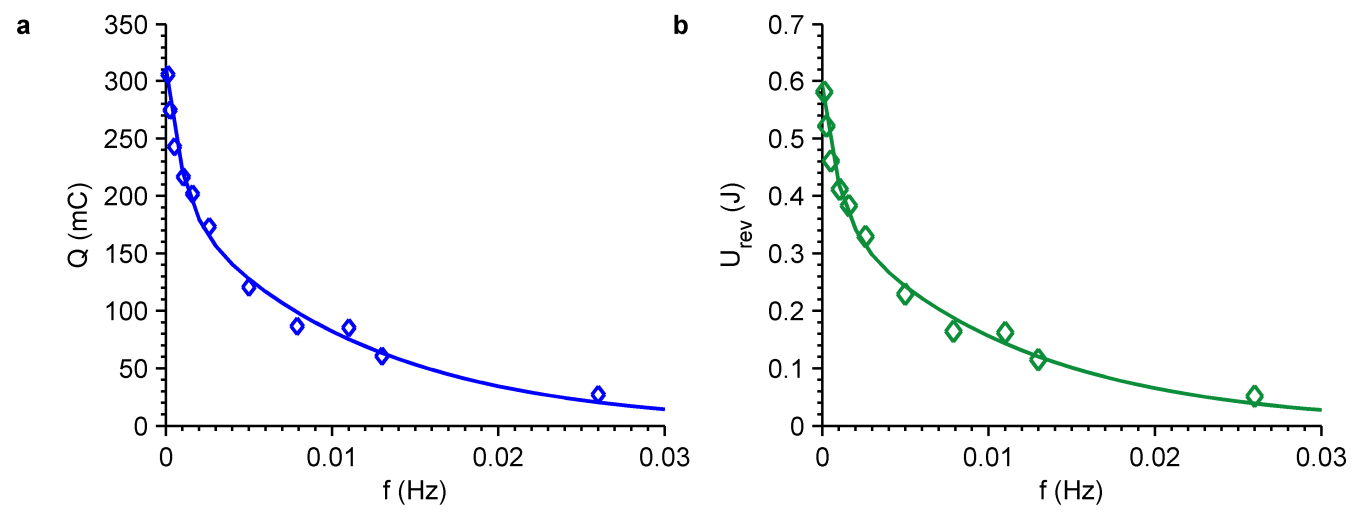

Figure 5 Charge (a) and energy (b) evolution of the PPy-DBS-MWCNT film in aqueous solution of $0.1 \mathrm{M}$ NaClO4

The empirical evolution of the consumed energy is adjusted by addition of two exponential functions of the frequency:

$$
Q_{r e v}(f)=a e^{b f}+c e^{d f}
$$

where $\mathrm{a}=115.4 \mathrm{mC}, \mathrm{b}=-1030 \mathrm{~s}, \mathrm{c}=196.2 \mathrm{mC}$ and $\mathrm{d}=-86.9 \mathrm{~s}$ and the correlation coefficient $\mathrm{r}^{2}=0.99$.

The effective reversible energy $U_{\text {rev }}$ consumed by the bilayer is determined from the consumed reversible charge $Q_{r e v}$ multiplied by the ratio of the studied potential range $\Delta E$. The potential is the potential energy power per unit charge.

$$
\begin{aligned}
& U_{\text {rev }}=Q_{\text {rev }}(f) \cdot \Delta E \\
& U_{\text {rev }}=\left(a e^{b f}+c e^{d f}\right) \cdot \Delta E
\end{aligned}
$$

Here the potential is constant and equals the potential range $\Delta E=1.9 \mathrm{~V}$, the difference between the sweep anodic and the cathodic potential limits.

The effective consumed reversible energy during reversible reduction and oxidation processes obtained from the empirical results at various frequencies is shown in Figure $5 \mathrm{~b}$. The maximum consumed reversible energy of the PPyDBS-MWCNT/tape bilayer in $0.1 \mathrm{M} \mathrm{NaClO}_{4}$ aqueous solution is obtained at the minimum scan rate $(\mathrm{f}=0)$, which means under the thermodynamic equilibrium.

$$
U_{\text {rev }}=(a+c) \cdot \Delta E=0.59 \mathrm{~J}
$$

The empirical results show that the effective consumed energy is a function of the frequency, which determines here the electrochemical energetic working conditions. Starting from the same initial reduced state a different oxidized state, requiring a different consumed charge and consuming a different electrical energy, is reached for each experimental frequency. In other words, the energy consumed by reactions during the reversible actuation of the PPy-DBS-MWCNT artificial muscle senses the available electrochemical energy (constant temperature, constant electrolyte concentration, constant potential range and different scan rates). Hence, the bilayer muscle senses during actuation the available electrochemical energy.

\section{CONCLUSION}

Increasing reversible angular displacements, up to $127^{\circ}$, and redox charges are attained at decreasing frequencies when the actuator is operated with triangular potential waves. The angular displacement is under control of the consumed charge being described by addition of two linear functions, Eq. 5 and 6 (this is a Faradaic motor) showing hysteresis effects. Each of the two functions is related to the electrochemical reaction of one of the two electroactive components: 
polypyrrole and MWCNT. The hysteresis of the muscle movement can be described by the Duhem model. The irreversible reaction overlapping the material reduction at high cathodic potentials originates some creeping effects: the muscle does not recover the initial position at the end of the potential cycle.

Either, the consumed charge and the consumed energy per cycle change with the frequency, following exponential functions. The muscle senses during actuation the applied electrochemical energy, being the attained exponential functions the sensing equations. It means that the electrochemical reactions of the muscle constitutive material (PPyDBS-MWCNT) drive both, the muscle actuation and the electrochemical sensor. This is a dual sensing-motor based on the simultaneous actuation and sensing properties of the constituent electrochemical molecular motors: the polypyrrole chains and the MWCNTs. The PPy-DBS-MWCNT/tape bilayer is a dual sensing-actuation muscle mimicking haptic vertebrate muscles.

\section{ACKNOWLEDGEMENT}

The research was supported by European Union's Horizon 2020 research and innovation program under the Marie Sklodowska-Curie grant agreement No 641822 .

\section{REFERENCES}

[1] Naka, Y., Fuchiwaki, M.., Tanaka, K., “A micropump driven by a polypyrrole based conducting polymer soft actuator," Polym. Int. 59(3), 352-356 (2010).

[2] Ramírez-García, S.., Diamond, D., "Biomimetic, low power pumps based on soft actuators," Sens. Actuators Phys. 135(1), 229-235 (2007).

[3] Tavakol, B., Bozlar, M., Punckt, C., Froehlicher, G., Stone, H. A., Aksay, I. A.., Holmes, D. P., "Buckling of dielectric elastomeric plates for soft, electrically active microfluidic pumps," Soft Matter 10(27), 4789-4794 (2014).

[4] Wu, Y., Zhou, D., Spinks, G. M., Innis, P. C., Megill, W. M.., Wallace, G. G., “TITAN: a conducting polymer based microfluidic pump," Smart Mater. Struct. 14(6), 1511 (2005).

[5] Giousouf, M.., Kovacs, G., "Dielectric elastomer actuators used for pneumatic valve technology," Smart Mater. Struct. 22(10), 104010 (2013).

[6] Karunanidhi, S.., Singaperumal, M., "Design, analysis and simulation of magnetostrictive actuator and its application to high dynamic servo valve," Sens. Actuators Phys. 157(2), 185-197 (2010).

[7] Moghadam, A. A. A., Kouzani, A., Torabi, K., Kaynak, A.., Shahinpoor, M., "Development of a novel soft parallel robot equipped with polymeric artificial muscles," Smart Mater. Struct. 24(3), 35017 (2015).

[8] Rus, D.., Tolley, M. T., "Design, fabrication and control of soft robots," Nature 521(7553), 467-475 (2015).

[9] Banerjee, K., Rajaeipour, P., Ataman, Ç.., Zappe, H., "Piezoelectric PVDF actuated, lightweight deformable thin mirror for adaptive optics," Opt. MEMS Nanophotonics OMN 2016 Int. Conf. On, 1-2, IEEE (2016).

[10] Shian, S., Diebold, R. M.., Clarke, D. R., "Tunable lenses using transparent dielectric elastomer actuators," Opt. Express 21(7), 8669-8676 (2013).

[11] Gisby, T. A., O’Brien, B. M.., Anderson, I. A., "Self sensing feedback for dielectric elastomer actuators," Appl. Phys. Lett. 102(19), 193703 (2013).

[12] Rosset, S., O’Brien, B. M., Gisby, T., Xu, D., Shea, H. R.., Anderson, I. A., "Self-sensing dielectric elastomer actuators in closed-loop operation," Smart Mater. Struct. 22(10), 104018 (2013).

[13] Jung, K., Kim, K. J.., Choi, H. R., “A self-sensing dielectric elastomer actuator,” Sens. Actuators Phys. 143(2), 343-351 (2008).

[14] Punning, A., Kruusmaa, M.., Aabloo, A., "A self-sensing ion conducting polymer metal composite (IPMC) actuator," Sens. Actuators Phys. 136(2), 656-664 (2007). 
[15] Sasaki, M., Lin, W., Tamagawa, H., Ito, S.., Kikuchi, K., "Self-sensing control of nafion-based ionic polymermetal composite (IPMC) actuator in the extremely low humidity environment," Actuators 2, 74-85, Multidisciplinary Digital Publishing Institute (2013).

[16] Lan, C.-C., Lin, C.-M.., Fan, C.-H., "A self-sensing microgripper module with wide handling ranges," IEEEASME Trans. Mechatron. 16(1), 141-150 (2011).

[17] Anderson, E. H.., Hagood, N. W., "Simultaneous piezoelectric sensing/actuation: analysis and application to controlled structures," J. Sound Vib. 174(5), 617-639 (1994).

[18] Dosch, J. J., Inman, D. J.., Garcia, E., “A self-sensing piezoelectric actuator for collocated control,” J. Intell. Mater. Syst. Struct. 3(1), 166-185 (1992).

[19] Rakotondrabe, M., Ivan, I. A., Khadraoui, S., Lutz, P.., Chaillet, N., "Simultaneous displacement/force selfsensing in piezoelectric actuators and applications to robust control," IEEEASME Trans. Mechatron. 20(2), 519531 (2015).

[20] Pratt, J.., Flatau, A. B., "Development and analysis of a self-sensing magnetostrictive actuator design," J. Intell. Mater. Syst. Struct. 6(5), 639-648 (1995).

[21] Martinez, J. G.., Otero, T. F., "Mechanical awareness from sensing artificial muscles: Experiments and modeling," Sens. Actuators B Chem. 195, 365-372 (2014).

[22] Otero, T. F.., Martinez, J. G., "Physical and chemical awareness from sensing polymeric artificial muscles. Experiments and modeling," Prog. Polym. Sci. 44, 62-78 (2015).

[23] Otero, T. F., Sanchez, J. J., Martinez, J. G., "Biomimetic dual sensing-actuators based on conducting polymers. Galvanostatic theoretical model for actuators sensing temperature," J. Phys. Chem. B 116(17), 5279-5290 (2012).

[24] Otero, T. F., Conducting Polymers, The Royal Society of Chemistry (2016).

[25] Martínez, J. G., Sugino, T., Asaka, K.., Otero, T. F., "Electrochemistry of Carbon Nanotubes: Reactive Processes, Dual Sensing-Actuating Properties and Devices," ChemPhysChem 13(8), 2108-2114 (2012).

[26] Li, C., Thostenson, E. T.., Chou, T.-W., "Sensors and actuators based on carbon nanotubes and their composites: A review," Compos. Sci. Technol. 68(6), 1227-1249 (2008).

[27] Martinez, J. G., Otero, T. F., Bosch-Navarro, C., Coronado, E., Marti-Gastaldo, C.., Prima-Garcia, H., "Graphene electrochemical responses sense surroundings," Electrochimica Acta 81, 49-57 (2012).

[28] Kiefer, R., Temmer, R., Tamm, T., Travas-Sejdic, J., Kilmartin, P. A.., Aabloo, A., "Conducting polymer actuators formed on MWCNT and PEDOT-PSS conductive coatings," Synth. Met. 171, 69-75 (2013).

[29] Sugino, T., Kiyohara, K., Takeuchi, I., Mukai, K.., Asaka, K., "Improving the actuating response of carbon nanotube/ionic liquid composites by the addition of conductive nanoparticles," Carbon 49(11), 3560-3570 (2011).

[30] Spinks, G. M., Xi, B., Truong, V.-T.., Wallace, G. G., "Actuation behaviour of layered composites of polyaniline, carbon nanotubes and polypyrrole," Synth. Met. 151(1), 85-91 (2005).

[31] Zheng, W., Razal, J. M., Whitten, P. G., Ovalle Robles, R., Wallace, G. G., Baughman, R. H., Spinks, G. M., "Artificial muscles based on polypyrrole/carbon nanotube laminates," Adv. Mater. 23(26), 2966-2970 (2011).

[32] Yun, S.., Kim, J., "Multiwalled-carbon nanotubes and polyaniline coating on electro-active paper for bending actuator," J. Phys. Appl. Phys. 39(12), 2580 (2006).

[33] Otero, T. F., Schumacher, J.., Pascual, V. H., "Construction and coulodynamic characterization of PPy-DBSMWCNT/tape bilayer artificial muscles," RSC Adv. 6(72), 68538-68544 (2016).

[34] Tahhan, M., Truong, V.-T., Spinks, G. M.., Wallace, G. G., "Carbon nanotube and polyaniline composite actuators*," Smart Mater. Struct. 12(4), 626 (2003).

[35] Otero, T. F.., Schumacher, J., "Electrochemical synthesis and characterization of self-supported polypyrroleDBS-MWCNT electrodes," J. Electroanal. Chem. 782, 182-191 (2016).

[36] Valero, L., Martinez, J. G.., Otero, T. F., "Creeping and structural effects in Faradaic artificial muscles," J. Solid State Electrochem. 19(9), 2683-2689 (2015).

[37] Fuchiwaki, M., Martinez, J. G.., Otero, T. F., "Asymmetric Bilayer Muscles. Cooperative and Antagonist Actuation," Electrochimica Acta 195, 9-18 (2016).

[38] Otero, T. F., Martinez, J. G.., Arias-Pardilla, J., "Biomimetic electrochemistry from conducting polymers. A review: artificial muscles, smart membranes, smart drug delivery and computer/neuron interfaces," Electrochimica Acta 84, 112-128 (2012). 
[39] Song, G., Zhao, J., Zhou, X.., De Abreu-García, J. A., “Tracking control of a piezoceramic actuator with hysteresis compensation using inverse Preisach model," IEEEASME Trans. Mechatron. 10(2), 198-209 (2005).

[40] Ge, P.., Jouaneh, M., "Generalized Preisach model for hysteresis nonlinearity of piezoceramic actuators," Precis. Eng. 20(2), 99-111 (1997).

[41] Dubra, A., Massa, J. S.., Paterson, C., "Preisach classical and nonlinear modeling of hysteresis in piezoceramic deformable mirrors," Opt. Express 13(22), 9062-9070 (2005).

[42] Al Janaideh, M., Rakheja, S.., Su, C.-Y., "An analytical generalized Prandtl-Ishlinskii model inversion for hysteresis compensation in micropositioning control," IEEEASME Trans. Mechatron. 16(4), 734-744 (2011).

[43] Gu, G.-Y., Zhu, L.-M.., Su, C.-Y., "Modeling and compensation of asymmetric hysteresis nonlinearity for piezoceramic actuators with a modified Prandtl-Ishlinskii model,” IEEE Trans. Ind. Electron. 61(3), 1583-1595 (2014).

[44] Liu, Y., Shan, J., Gabbert, U.., Qi, N., "Hysteresis and creep modeling and compensation for a piezoelectric actuator using a fractional-order Maxwell resistive capacitor approach," Smart Mater. Struct. 22(11), 115020 (2013).

[45] Goldfarb, M.., Celanovic, N., "Modeling piezoelectric stack actuators for control of micromanipulation,” IEEE Control Syst. Mag. 17(3), 69-79 (1997).

[46] Wang, X., Alici, G.., Tan, X., "Modeling and inverse feedforward control for conducting polymer actuators with hysteresis," Smart Mater. Struct. 23(2), 25015 (2013). 